\title{
MATERIALS FOR INNOVATION
}
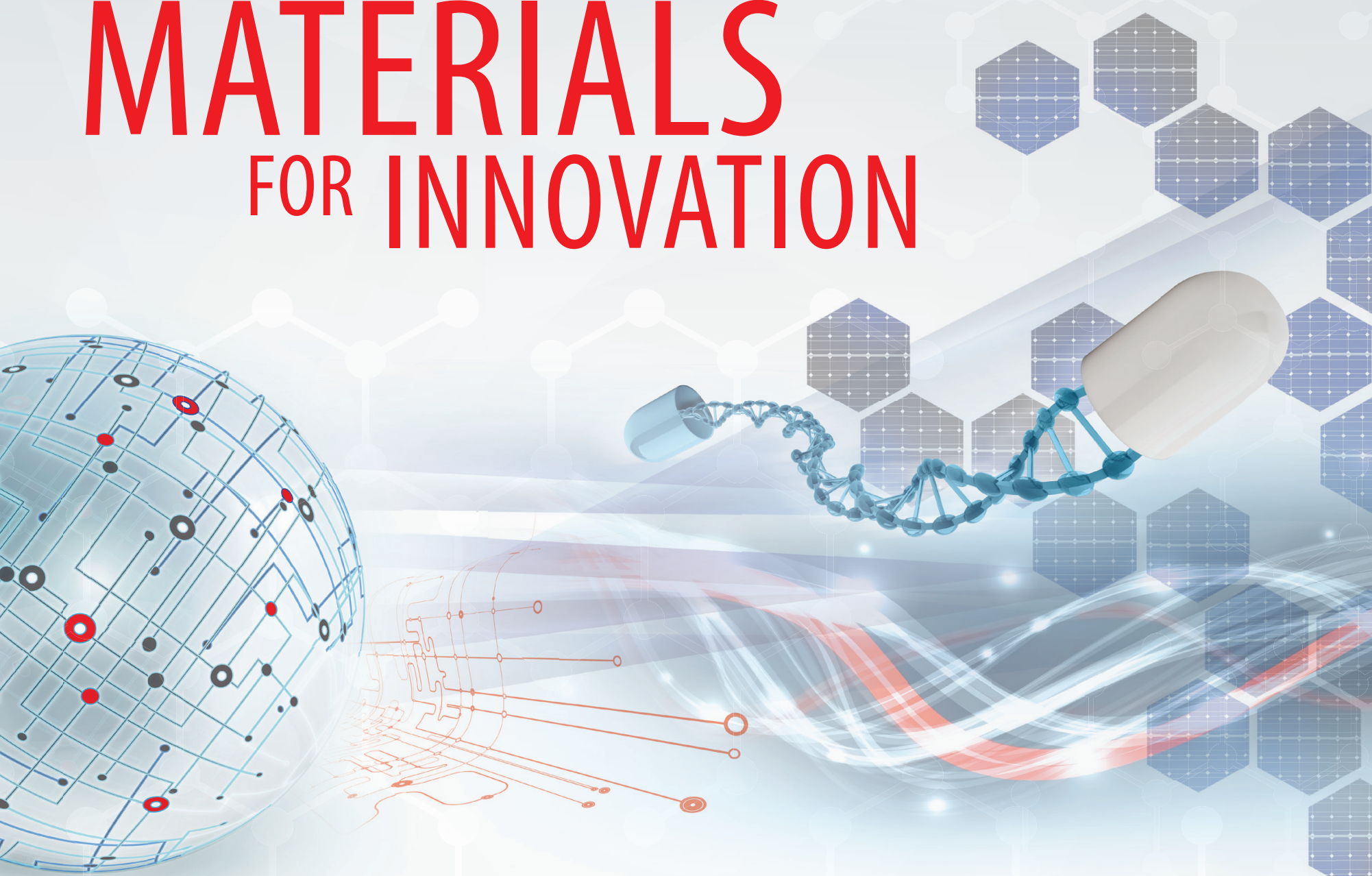

\section{ENERGY}

\section{BIOMEDICAL}

Materials for drug delivery, tissue engineering, and regenerative medicine; PEGs, biodegradable and natural polymers; functionalized nanoparticles; block copolymers, dendrimers and nanoclays

\section{ELECTRONICS}

Nanowires; printed electronics inks and pastes; materials for OPV, OFET, OLED; nanodispersions; CNTs and graphene; precursors for PVD, CVD, and sputtering
Ready-to-use battery grade electrode and electrolyte materials; nanopowders, nanostructures and dispersions; quantum dots; perovskites; fuel cells and membrane; hydrogen storage materials including MOFs; phosphors; thermoelectrics; high purity salts

Find more information on our capabilities at aldrich.com/matsci 
dysprosium pellets atomic layer deposition scandium-aluminum

\begin{tabular}{|c|c|c|c|c|c|c|c|c|c|c|c|c|c|c|c|c|c|}
\hline Li & Be & & & & & & & & & & & B & C & $\mathbf{N}$ & 0 & $\mathbf{F}$ & $\mathrm{Ne}$ \\
\hline $\mathrm{Na}$ & $\mathbf{M g}$ & & en & cor & UC & Drs & & alla & diun & sh & & AI & Si & $\mathbf{P}$ & $\mathbf{S}$ & CI & Ar \\
\hline $\mathbf{K}$ & Ca & Sc & $\mathbf{T i}$ & $\mathbf{V}$ & $\mathrm{Cr}$ & Mn & $\mathbf{F e}$ & Co & $\mathbf{N i}$ & Cu & $\mathbf{Z n}$ & $\mathbf{G a}$ & Ge & As & Se & $\mathbf{B r}$ & $\mathbf{K r}$ \\
\hline $\mathbf{R b}$ & Sr & $\mathbf{Y}$ & $\mathbf{Z r}$ & $\mathbf{N b}$ & Mo & Tc & $\mathbf{R u}$ & $\mathbf{R h}$ & Pd & $\mathbf{A g}$ & Cd & In & Sn & $\mathbf{S b}$ & Te & I & Xe \\
\hline Cs & $\mathbf{B a}$ & La & Hf & Ta & $\mathbf{W}$ & Re & Os & Ir & Pt & $\mathbf{A u}$ & Hg & TI & $\mathbf{P b}$ & $\mathbf{B i}$ & Po & $\mathbf{A t}$ & Rn \\
\hline Fr & $\mathbf{R a}$ & Ac & Rf & Db & Sg & $\mathbf{B h}$ & Hs & $\mathbf{M t}$ & Ds & $\mathbf{R g}$ & Cn & Uut & $\mathbf{F I}$ & Uup & Lv & Uus & Uno \\
\hline
\end{tabular}

\begin{tabular}{|c|c|c|c|c|c|c|c|c|c|c|c|c|c|c|}
\hline dielectrics & Ce & Pr & Nd & Pm & Sm & Eu & Gd & Tb & Dy & Ho & Er & Tm & $\mathbf{Y b}$ & Lu \\
\hline & Th & $\mathbf{P a}$ & $\mathbf{U}$ & $\mathbf{N p}$ & $\mathbf{P u}$ & Am & $\mathrm{Cm}$ & Bk & Cf & Es & Fm & Md & No & Lr \\
\hline
\end{tabular}

super alloys nanofabrics rare earth metals nickelfoam LED lighting

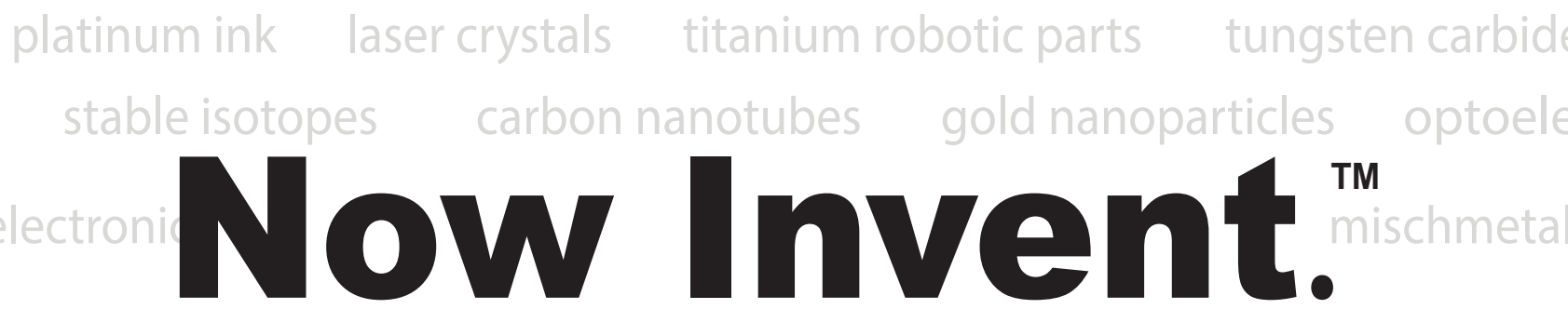

anti-ballistic ceramics fuel cellmaterials hafnium tubing Nd:YAG

germanium windows superconductors

99.999\% ruthenium spheres

erbium doped fiber optics 